\title{
A SINGULAR INTEGRAL
}

\author{
JAVAD NAMAZI ${ }^{1}$
}

\begin{abstract}
In this paper we show that if $K(x)=\Omega(x) /|x|^{n}$ is a CalderónZygmund kernel, where $\Omega \in L^{q}\left(S^{n-1}\right)$ for some $1<q \leq \infty$, and $b$ is a radial bounded function, then $b(x) K(x)$ is the kernel of a convolution operator which is bounded on $L^{p}\left(R^{n}\right)$ for $1<p<\infty$ and $n \geq 2$.
\end{abstract}

This paper is related to boundedness properties of a variation of CalderónZygmund operators.

Let $H(x)=b(|x|) \Omega(x) /|x|^{n}$ be a kernel, where $\int_{S^{n-1}} \Omega(x) d \sigma\left(x^{\prime}\right)=0, \Omega(\lambda x)=$ $\Omega(x)$ for $x \in R^{n}, \lambda>0$, and $b$ is radial. Define

$$
f(x)=\lim _{\varepsilon \rightarrow 0} \int_{|y|>\varepsilon} H(y) f(x-y) d y=\text { P.V. } H * f(x) .
$$

If $b \equiv 1$ and $\Omega \in L^{q}\left(S^{n-1}\right)$ for some $q>1$, then $T$ is a Calderón-Zygmund operator which is bounded on $L^{p}\left(R^{n}\right)$ for $1<p<\infty$ and $n \geq 1$; see [4].

In [1] R. Fefferman showed that if $\Omega$ satisfies a Lipschitz condition and $b$ is bounded, then $T$ is bounded on $L^{p}\left(R^{n}\right), 1<p<\infty, n \geq 2$. This is not true when $n=1$; for instance, if $H(x)=\sin (|x|) / x$, then its Fourier transform $\hat{H}(\xi)$ is unbounded, indicating that $T$ cannot be bounded on $L^{2}\left(R^{1}\right)$. It turns out that the smoothness condition on $\Omega$ can be relaxed a great deal.

THEOREM. If $\Omega \in L^{q}\left(S^{n-1}\right)$ for some $1<q \leq \infty$, and $b(|x|) \in L^{\infty}$, then $T$ is bounded on $L^{p}\left(R^{n}\right)$ for $1<p<\infty$ and $n \geq 2$.

ProOF. First we show that the Fourier transform of $H$ is bounded; thereby $T$ is bounded on $L^{2}\left(R^{n}\right)$. Using polar coordinates and letting $x=\rho x^{\prime}, \rho=|x|$, we get

$$
\begin{aligned}
\hat{H}(\xi) & =\lim _{\varepsilon \rightarrow 0} \int_{|x|>\varepsilon} \frac{b(|x|)}{|x|^{n}} \Omega(x) e^{-i x \cdot \xi} d x \\
& =\lim _{\varepsilon \rightarrow 0} \int_{\varepsilon<\rho<1 / \varepsilon} \frac{b(\rho)}{\rho} d \rho\left(\int_{S^{n-1}} \Omega\left(x^{\prime}\right) e^{i \rho x^{\prime} \cdot \xi} d \sigma\left(x^{\prime}\right)\right) .
\end{aligned}
$$

By a change of variable $r=\rho|\xi|,(2)$ becomes

$$
\begin{aligned}
\hat{H}(\xi) & =\lim _{\varepsilon \rightarrow 0} \int_{\varepsilon|\xi|}^{|\xi| / \varepsilon} \frac{b(r /|\xi|)}{r} d r\left(\int_{S^{n-1}} \Omega\left(x^{\prime}\right) e^{-i r x^{\prime} \cdot \xi^{\prime}} d \sigma\left(x^{\prime}\right)\right) \\
& =\lim _{\varepsilon \rightarrow 0} \int_{\varepsilon|\xi|}^{|\xi| / \varepsilon} \frac{b(r /|\xi|)}{r} g\left(r, \xi^{\prime}\right) d r
\end{aligned}
$$

Received by the editors March 19, 1985.

1980 Mathematics Subject Classification. Primary 42B20.

Key words and phrases. Calderón-Zygmund kernels.

${ }^{1}$ This work is part of the author's dissertation completed at Indiana University under the supervision of Alberto Torchinsky. 
where

Now if

$$
g\left(r, \xi^{\prime}\right)=\int_{S^{n-1}} \Omega\left(x^{\prime}\right) e^{-i r x^{\prime} \cdot \xi^{\prime}} d \sigma\left(x^{\prime}\right), \quad\left|\xi^{\prime}\right|=1
$$

$$
\operatorname{Sup}_{\left|\xi^{\prime}\right|=1}\left\|g\left(\cdot, \xi^{\prime}\right)\right\|_{L^{p}(1, \infty)} \leq A
$$

for some $1<p<\infty$ and a constant $A$, then we claim that $\hat{H}(\xi)$ is bounded. To prove the claim, first we observe that

$$
\begin{aligned}
\left|g\left(r, \xi^{\prime}\right)\right| & =\left|\int_{S^{n-1}} \Omega\left(x^{\prime}\right) e^{-i r x^{\prime} \cdot \xi^{\prime}} d \sigma\left(x^{\prime}\right)\right| \\
& =\left|\int_{S^{n-1}} \Omega\left(x^{\prime}\right)\left(e^{-i r x^{\prime} \cdot \xi^{\prime}}-1\right) d \sigma\left(x^{\prime}\right)\right| \\
& \leq 2 r\|\Omega\|_{L^{1}\left(S^{n-1}\right)}=c r
\end{aligned}
$$

by the mean value theorem. Using this and Hölder's inequality, we see that

$$
\begin{aligned}
\int_{0}^{\infty}\left|\frac{b(r /|\xi|)}{r} g\left(r, \xi^{\prime}\right)\right| d r & \leq\|b\|_{\infty} \int_{0}^{\infty} \frac{\left|g\left(r, \xi^{\prime}\right)\right|}{r} d r \\
& \leq\|b\|_{\infty}\left(\int_{0}^{1} \frac{c r}{r} d r+\left\|g\left(\cdot, \xi^{\prime}\right)\right\|_{L^{p}(1, \infty)} \cdot\left\|\frac{1}{r}\right\|_{L^{p^{\prime}(1, \infty)}}\right) \\
& \leq\|b\|_{\infty}\left(c+A\|1 / r\|_{\left.L^{p^{\prime}(1, \infty)}\right) \leq c}\right.
\end{aligned}
$$

where $1 / p^{\prime}=1-1 / p$. Hence, by Lebesgue's dominated convergence theorem, the limit in $(3)$ exists and $|\hat{H}(\xi)| \leq c$.

Now let us see when condition (4) may hold. Let $\rho$ be a rotation such that $\rho\left(\xi^{\prime}\right)=(1,0,0, \ldots, 0)$. In the integral of $g\left(r, \xi^{\prime}\right)$ make the change of variable $x^{\prime} \rightarrow$ $\rho^{-1} x^{\prime}$, and let $\Omega^{\rho}\left(x^{\prime}\right)=\Omega\left(\rho^{-1} x^{\prime}\right)$. Then

$$
\begin{aligned}
g\left(r, \xi^{\prime}\right) & =\int_{S^{n-1}} \Omega\left(x^{\prime}\right) e^{-i r x^{\prime} \cdot \xi^{\prime}} d \sigma\left(x^{\prime}\right)=\int_{S^{n-1}} \Omega^{\rho}\left(x^{\prime}\right) e^{-i r\left(\rho^{-1} x^{\prime}\right) \cdot \xi^{\prime}} d \sigma\left(x^{\prime}\right) \\
& =\int_{S^{n-1}} \Omega^{\rho}\left(x^{\prime}\right) e^{-i r x^{\prime} \cdot \rho \xi^{\prime}}=\int_{S^{n-1}} \Omega^{\rho}\left(x^{\prime}\right) e^{-i r x_{1}^{\prime}} d \sigma\left(x^{\prime}\right)
\end{aligned}
$$

where $x^{\prime}=\left(x_{1}^{\prime}, x_{2}^{\prime}, \ldots, x_{n}^{\prime}\right)=\left(x_{1}^{\prime}, x^{\prime \prime}\right)$. Taking $x_{1}^{\prime}=\cos \theta$, we can write the last integral as

$$
g\left(r, \xi^{\prime}\right)=\int_{0}^{\pi} e^{-i r \cos \theta} d \theta\left(\int_{S_{\cos \theta}} \Omega^{\rho}\left(\cos \theta, x^{\prime \prime}\right) d \sigma_{\theta}\left(x^{\prime}\right)\right)
$$

where $S_{\cos \theta}=\left\{\left|x^{\prime}\right|=1: x^{\prime}=\left(\cos \theta, x^{\prime \prime}\right)\right\}$ and $d \sigma_{\theta}\left(x^{\prime}\right)$ is its surface measure. By a further change of variable $s=\cos \theta$, the last integral is reduced to

$$
g\left(r, \xi^{\prime}\right)=\int_{-1}^{1} e^{-i r s}\left(1-s^{2}\right)^{-1 / 2}\left(\int_{S_{s}} \Omega^{\rho}\left(s, x^{\prime \prime}\right) d \sigma_{s}\left(x^{\prime}\right)\right) d s .
$$

Set $x^{\prime \prime}=\sqrt{1-s^{2}} y^{\prime}$. Then $y^{\prime} \in S^{n-2}$ and

$$
g\left(r, \xi^{\prime}\right)=\int_{-1}^{1} e^{-i r s}\left(1-s^{2}\right)^{(n-3) / 2}\left(\int_{S^{n-2}} \Omega^{\rho}\left(s, \sqrt{1-s^{2}} y^{\prime}\right) d \sigma_{n-2}\left(y^{\prime}\right)\right)
$$

where $d \sigma_{n-2}\left(y^{\prime}\right)$ is the surface measure of $S^{n-2}$. 
From (5) we observe that $g\left(r, \xi^{\prime}\right)=(h(s) w(s))^{\uparrow}(r)$, where

$h(s)=\int_{S^{n-2}} \Omega^{\rho}\left(s, \sqrt{1-s^{2}} y^{\prime}\right) d \sigma_{n-2}\left(y^{\prime}\right) \quad$ and $\quad w(s)=\left(1-s^{2}\right)^{(n-3) / 2} \chi_{(-1,1)}(s)$.

Without loss of generality, we may assume that $\Omega \in L^{q}\left(S^{n-1}\right)$ for some $1<q<$ $\infty$. Let $q^{\prime}$ be the conjugate of $q ; 1 / q^{\prime}+1 / q=1$. By Hölder's inequality,

$$
\begin{aligned}
|h(s)| & =\left|\int_{S^{n-2}} \Omega^{\rho}\left(s, \sqrt{1-s^{2}} y^{\prime}\right) d \sigma_{n-2}\left(y^{\prime}\right)\right| \\
& \leq \omega_{n-2}^{1 / q^{\prime}}\left(\int_{S^{n-2}}\left|\Omega^{\rho}\left(s, \sqrt{1-s^{2}} y^{\prime}\right)\right|^{q} d \sigma_{n-2}\left(y^{\prime}\right)\right)^{1 / q} \\
& =c\left(\int\left|\Omega^{\rho}\left(s, \sqrt{1-s^{2}} y^{\prime}\right)\right|^{q} d \sigma_{n-2}\left(y^{\prime}\right)\right)^{1 / q} .
\end{aligned}
$$

$\omega_{n-2}$ is the area of $S^{n-2}$. Hence,

$$
\begin{aligned}
\int_{-1}^{1}|h(s)|^{q} w(s) d s & \leq c \int_{-1}^{1}\left(1-s^{2}\right)^{(n-3) / 2} d s \int_{S^{n-2}}\left|\Omega^{\rho}\left(s, \sqrt{1-s^{2}} y^{\prime}\right)\right|^{q} d \sigma_{n-2}\left(y^{\prime}\right) \\
& =c\|\Omega\|_{L^{q}\left(S^{n-1}\right)}^{q} .
\end{aligned}
$$

Therefore, $|h(s)|^{q} w(s) \in L^{1}(-1,1)$.

Let $1<p^{\prime}<q$ and $r=q / p^{\prime}$. Using Hölder's inequality again,

$$
\begin{aligned}
\int_{-1}^{1}|h(s) w(s)|^{p^{\prime}} d s & =\int_{-1}^{1}|h(s)|^{p^{\prime}} w(s)^{1 / r} w(s)^{p^{\prime}-1 / r} d s \\
& \leq\left(\int_{-1}^{1}|h(s)|^{q} w(s) d s\right)^{1 / r}\left(\int_{-1}^{1} w(s)^{r^{\prime}\left(p^{\prime}-1 / r\right)} d s\right)^{1 / r^{\prime}} \\
& \leq c\|\Omega\|_{L^{q}\left(S^{n-1}\right)}^{q / r}\left(\int_{-1}^{1}\left(1-s^{2}\right)^{[(n-3) / 2] r^{\prime}\left(p^{\prime}-1 / r\right)} d s\right)^{1 / r^{\prime}} \\
& =c\|\Omega\|_{L^{q}\left(S^{\prime}-1\right)}^{p^{\prime}}\left(\int_{-\pi / 2}^{\pi / 2}|\sin \theta|^{(n-3) r^{\prime}\left(p^{\prime}-1 / r\right)+1} d \theta\right)^{1 / r^{\prime}}
\end{aligned}
$$

This last integral is convergent if $(n-3) r^{\prime}\left(p^{\prime}-1 / r\right)+1>-1$. This inequality, obviously, holds for all $p^{\prime}$ when $n \geq 3$. In the case $n=2$, it holds whenever $1<p^{\prime}<2 /(1+1 / q)$. Therefore, if $\Omega \in L^{q}\left(S^{n-1}\right), 1<q \leq \infty$, then there always exists some $p^{\prime}, 1<p^{\prime}<2$, such that $h(s) w(s) \in L^{p^{\prime}}(-1,1)$. By the HausdorffYoung inequality

$$
\begin{aligned}
\left\|g\left(\cdot, \xi^{\prime}\right)\right\|_{L^{p}(1, \infty)} & \leq\left\|g\left(\cdot, \xi^{\prime}\right)\right\|_{L^{p}(-\infty, \infty)}=\left\|(h(s) w(s))^{-}\right\|_{L^{p}(-\infty, \infty)} \\
& \leq\|h(s) w(s)\|_{L^{p^{\prime}}(-\infty, \infty)} \\
& =\|h(s) w(s)\|_{L^{p^{\prime}}(-1,1)} \leq c\|\Omega\|_{L^{q}\left(S^{n-1}\right)}^{p^{\prime}}=A .
\end{aligned}
$$

This proves that $\hat{H}(\xi)$ is bounded, and thus $T$ is bounded on $L^{2}\left(R^{n}\right), n \geq 2$.

Using the technique of complex interpolation and an argument similar to the one in $[\mathbf{1}]$, the $L^{p}$ boundedness can be established. We mention it briefly. 
Define

and

$$
m_{z}(\xi)=\mathrm{P} . \mathrm{V} . \int_{R^{n}} \frac{H(x)}{|x|^{z}} e^{-i x \cdot \xi} d x \cdot|\xi|^{-z}
$$

$$
\left(T_{z} f\right)^{-}(\xi)=m_{z}(\xi) \hat{f}(\xi),
$$

$|\operatorname{Re}(z)|<\eta$ for some $\eta>0 . T_{z}$ is an analytic family of operators in the sense of Stein. Also $T_{0}=T$. Each $T_{z}$ is a principal-valued convolution operator, $T_{z} f=$ P.V. $H_{z} * f$, where $H_{z}=m_{z}$. As proved in [1], if $0<\operatorname{Re}(z) \leq \eta$ and $\eta$ small enough, then

$$
\sup _{|y|>0} \int_{|x|>2|y|}\left|H_{z}(x+y)-H_{z}(x)\right| d x \leq c_{z},
$$

where the constant $c_{z}$ depends only on the real part of $z$. Also a similar argument as in the $L^{2}$ case will show that $\left|m_{z}(\xi)\right| \leq c$ for $|\operatorname{Re}(z)| \leq \eta$, when $\eta$ is small enough. Therefore $\left\|T_{-\eta+i y}\right\|_{2} \leq c$ and $\left\|T_{\eta+i y}\right\|_{r} \leq c_{\eta}$ for $-\infty<y<\infty$ and $1<r<\infty$. By complex interpolation, if $1 / p=(1-1 / 2) / 2+(1 / 2) / r$ then $\|T\|_{p}=\left\|T_{0}\right\|_{p} \leq c$. Hence, $T$ is bounded on $L^{p}\left(R^{n}\right), 1<p<\infty, n \geq 2$.

\section{BIBLIOGRAPHY}

1. Robert Fefferman, A note on a singular integral, Proc. Amer. Math. Soc. 74 (1979), 266270.

2. Javad Namazi, On a singular integral, Thesis reprint.

3. Elias M. Stein, Singular integrals and differentiability properties of functions, Princeton Univ. Press, Princeton, N.J., 1970.

4. Elias M. Stein and Guido Weiss, Introduction to Fourier analysis on Euclidean spaces, Princeton Univ. Press, Princeton, N.J., 1971.

Department of Mathematical and Computer Sciences, Michigan TechnoLOGICAL UNIVERSITY, HOUGHTON, MICHIGAN 49931 